\title{
Removal of Ibuprofen From Synthetic Wastewater Using Photocatalytic Method in the Presence of Feo Photocatalyst Supported on Modified Iranian Clinoptilolite
}

Majid Mohadesi ( $\sim$ m.mohadesi@gmail.com )

Kermanshah University of Technology https://orcid.org/0000-0003-1944-6435

Ashkan Gouran

Kermanshah University of Technology

Kiarash Seifi

Kermanshah University of Technology

\section{Research Article}

Keywords: Ibuprofen, Photocatalytic process, Clinoptilolite, Iron oxide, Photocatalyst recovery

Posted Date: August 30th, 2021

DOl: https://doi.org/10.21203/rs.3.rs-582241/v1

License: (c) (1) This work is licensed under a Creative Commons Attribution 4.0 International License. Read Full License

Version of Record: A version of this preprint was published at Environmental Science and Pollution Research on January 17th, 2022. See the published version at https://doi.org/10.1007/s11356-02118153-3. 


\section{Abstract}

This study investigated the removal of an organic drug called ibuprofen from the wastewater containing this drug. Iron oxide supported on modified Iranian clinoptilolite was used as the photocatalyst in the presence of the light of a solar lamp. XRD, SEM, EDAX and FT-IR analyses were performed to detect the prepared photocatalyst. The results of photocatalytic identification analyses proved the suitable loading of iron oxide supported on modified Iranian clinoptilolite. This study investigated the effect of initial concentration of ibuprofen (5-25 mg/L), photocatalyst concentration (100-300 mg/L), and process time (10-240 $\mathrm{min}$ ) on the removal from ibuprofen from wastewater containing this drug. The experiments were performed in a setup in the presence of a solar lamp with a flux of $300 \mathrm{~W} / \mathrm{m}^{2}$. The results indicated that with the initial ibuprofen concentration of $25 \mathrm{mg} / \mathrm{L}$, photocatalyst concentration of $300 \mathrm{mg} / \mathrm{L}$, and time of $210 \mathrm{~min}$, the highest percentage of ibuprofen removal was $99.80 \%$. Kinetic modeling was then performed using the Langmuir-Hinshelwood model, and a quasi-first-order kinetic model showed a good agreement with the results obtained. Finally, the recovery of the photocatalyst was investigated and the results showed that under optimal conditions about $91 \%$ of ibuprofen was removed after five re-uses of the photocatalyst.

\section{Introduction}

Access to clean water is the main problem of human. The shortage of drinking water in many parts of the world has turn into an emergent concern in recent years. The evolution of new technologies and industrial processes has been associated with an increase in the use of chemicals to produce new products. Water resources are often contaminated through the entry of common contaminants including painting materials, heavy metals, as well as microorganisms that cause various diseases (Gopinath et al. 2020).

About 9,000 chemical compounds are currently used for pharmaceutical applications worldwide. These compounds generally form a diverse range of chemical structures (Arthur et al. 2018). During the last few decades, drugs have been considered as one of the most important groups of water pollutants (Apopei et al. 2020). These pollutants include other items such as pesticides and personal care products and illegal incendiary substances, food supplements, etc. This type of contaminants is increasingly being produced in the pharmaceutical industry, and every concentration of these emerging contaminants in aqueous media (from micrograms to nanograms per liter) can affect aquatic organisms and human health (Candido et al. 2016).

Ibuprofen (IBU) is one of the most widely used drugs, which is slightly soluble in water, but is soluble in most organic solvents (Gu et al. 2019). This anti-inflammatory drug is used to treat menstrual pain and inflammation worldwide (Zhou et al. 2020). Ibuprofen has the potential to cause gastrointestinal bleeding and heart diseases.

Hence, it is essential to utilize an effective method to treat this drug. There are several methods for removing ibuprofen, including the use of the adsorption process, activated carbon, microbial biofilters, 
membranes-based biological or physical processes, and advanced oxidation processes (Kang et al. 2014). Heterogeneous photocatalytic process is one of the most important studied methods; this method has several advantages including environmental protection, energy conservation, and cost-effectiveness (Sarafraz et al. 2020).

Because of the high applicability of Zeolite, it has been known investigated in many studies for over 200 years. It has several advantages including high ion exchange capability and material absorption and low cost; moreover, it is mostly used in the field of energy generation for pollution control and adsorption (Aghel et al. 2020). Environmental problems caused due to the consumption of traditional fossil fuels also encourage the development of renewable energy sources. Among the various types of clean energy, solar energy has received much attention. Solar energy has advantages such as high capacity and generality, though its decentralized nature is still a major challenge hindering practical applications (Zhang et al. 2020). Taking the mentioned items into consideration, a number of studies have been conducted on the removal of ibuprofen via photocatalytic methods.

In a study, a new $\mathrm{Bi}_{2} \mathrm{O}_{4} / \mathrm{Fe}_{3} \mathrm{O}_{4}$ superparamagnetic nanocomposite was successfully prepared using the in-situ growth method and used for the photocatalytic removal of ibuprofen. $\mathrm{Bi}_{2} \mathrm{O}_{4} / \mathrm{Fe}_{3} \mathrm{O}_{4}$ (2.5:1) was magnetically recycled and demonstrated good reusability without significant loss of photocatalytic activity or structural change even after five cycles of reuse, which is promising for photocatalytic degradation of medicinal and hygienic contaminants from water (Xia and Lo 2016). A group of researchers investigated the mechanisms of photocatalytic degradation of ibuprofen via $\mathrm{BiVO}_{4}$ in aqueous media. The results showed that the prepared photocatalyst corresponded to the pure monoclinic scheelite of BiVO4 phase. With increasing the initial IBU concentration, the rate of photocatalytic degradation of ibuprofen decreased. The degradation process followed the first-order kinetic model (Li et al. 2016). Another study investigated the degradation of ibuprofen using diodes emitting ultraviolet light (UV-LEDs) in a $\mathrm{TiO}_{2}$ photocatalyst. Using a $\mathrm{TiO}_{2} / \mathrm{UV}$-LED system, the experiments were conducted on examples of ultra-pure water (UP) and secondary treated wastewater of a municipal wastewater treatment plant, both with IBU, as well as with a highly concentrated IBU $(230 \mathrm{mg} / \mathrm{L})$ obtained from pharmaceutical wastewater. The results indicated that the system was very efficient in removing this contaminant (Jallouli et al. 2018). In another study, BiOI microspheres were doped with different amounts of $\mathrm{Ti}$ and used to remove diclofenac from water under visible light. The results showed that Doped BiOI microspheres could be used as a cost-effective and efficient material for the effective degradation of newly growing pollutants (e.g. pharmaceuticals) from wastewater under light conditions (Liu et al. 2019). In a study, researchers investigated the adsorption and photocatalytic activity of titanium dioxide $\left(\mathrm{TiO}_{2}\right)$ boron nitride $(\mathrm{BN})$ nanocomposite to remove new ibuprofen contaminants in water as a model compound. $\mathrm{TiO}_{2}$ nanofibers wrapped by $\mathrm{BN}$ nanofibers were synthesized using electrospinning synthesis method. The experimental results showed that photocatalytic oxidation using TiO2-BN nanocomposites was a multi-step process and the interactions between ibuprofen molecules and TiO2-BN nanocomposites controlled the adsorption process (Lin et al. 2019). In one study, a heterogeneous Zdesign g- $\mathrm{C}_{3} \mathrm{~N}_{4} / \mathrm{TiO}_{2} / \mathrm{Fe}_{3} \mathrm{O}_{4} @ \mathrm{SiO}_{2}$ (gCTFS) heterogeneous nanophotocatalyst with high magnetic 
recyclability was synthesized through the sol-gel method using only a small amount of g- $\mathrm{C}_{3} \mathrm{~N}_{4}$ ultra-thin nanosheets. This nanophotocatalyst was able to remove $97 \%$ of ibuprofen from visible light after 15 minutes of continuous irradiation of $330 \mathrm{~W} / \mathrm{m}^{2}$ (Kumar et al. 2018). A group of researchers studied the degradation of an anti-inflammatory and antipyretic drug (ibuprofen) using advanced photocatalystbased oxidation processes. Catalysts $\left(\mathrm{TiO}_{2}\right.$ and $\left.\mathrm{ZnO}\right)$ were used together with artificial ultraviolet and solar radiation to produce highly oxidizing species, which led to the degradation of IBP into intermediates and eventually to carbon dioxide and water (Tanveer et al. 2019). Another study examined the removal of ibuprofen using activated carbon impregnated with $\mathrm{TiO}_{2}$. In the mentioned study, the emphasis was on the effect of various parameters, such as the type of composite, initial concentration of ibuprofen (5-25 $\mathrm{mg} / \mathrm{L})$, temperature $\left(22-28^{\circ} \mathrm{C}\right)$, and $\mathrm{pH}$ (acidic and alkaline solution). The experiment was performed in a tubular current reactor with a monochromatic $15 \mathrm{~W}$ lamp $(254 \mathrm{~nm})$. Due to the synergy of light absorption and degradation, AC90T10 composite provided the highest removal rate of ibuprofen solution (92\%) under UV light during $4 \mathrm{~h}$ (Gu et al. 2019).

A study examined the simultaneous production of hydrogen and the decomposition of ibuprofen using a homogeneous photocatalyst, which was made by adding Au nanoparticles to the surface of a batch of $\mathrm{TiO}_{2}$ mesocrystals to improve material properties. Using this method, effective separation was achieved, which was essential for redox reactions; this method creates new opportunities for the development of energy efficient methods for the degradation of this type of pollutants (Yao et al. 2020).

In this study, iron oxide/modified Iranian clinoptilolite (FeO/MIC) was used as a photocatalyst to remove ibuprofen from aqueous solution in an open channel under solar lamp light. The effect of various operational variables such as initial concentration of ibuprofen, photocatalyst concentration (FeO/MIC), and process time were investigated. The results indicated the high capacity of $\mathrm{FeO} / \mathrm{MIC}$ in the photocatalytic process of ibuprofen removal. In addition, in this study, a quasi-first-order kinetic model for the ibuprofen removal reaction in the presence of a photocatalyst was investigated. The results indicated the high accuracy of the kinetic model.

\section{Materials And Methods}

\subsection{Materials}

Iranian clinoptilolite zeolite (Semnan, Iran) was obtained from Afrand Tosca Company. Moreover, ferrous sulfate heptahydrate $\left(\mathrm{FeSO}_{4} \cdot 7 \mathrm{H}_{2} \mathrm{O}\right)$ obtained from Merck (>99\%) was used to coat the photocatalyst layer and ibuprofen obtained from Merck (>99\%) was used to make a synthetic solution of drug contaminant. The composition and physicochemical properties of this substance (ibuprofen) are presented in Table 1. Chloric acid obtained from Merck ( $>99 \%$ ) was used to modify the zeolite and $\mathrm{NaOH}$ obtained from Merck (> 99\%) was used to neutralize the zeolite. Deionized water was used in all stages of the experiment.

\subsection{Preparation of FeO/MIC photocatalyst}


In this study, iron oxide supported on modified Iranian clinoptilolite was used as the photocatalyst. First, in order to improve the properties of Iranian clinoptilolite (increasing the specific surface area and reducing the particle size), consistent with a research by Aghel et al. ( Aghel et al. 2020), the necessary modifications were made. Then, using $\mathrm{FeSO}_{4} \cdot 7 \mathrm{H}_{2} \mathrm{O}$, the modified Iranian clinoptilolite developed photocatalytic properties. Accordingly, for every $20 \mathrm{~g}$ of modified Iranian clinoptilolite, $200 \mathrm{~mL}$ of a solution of $0.2 \mathrm{~m}$ of ferrous sulfate heptahydrate was added and the mixture was stirred for $24 \mathrm{~h}$ at ambient temperature at $400 \mathrm{rpm}$. After filtration, the sample was washed with water and then dried at 120 ${ }^{\circ} \mathrm{C}$ for $24 \mathrm{~h}$, and finally calcined at $500{ }^{\circ} \mathrm{C}$ for $2 \mathrm{~h}$.

\subsection{Method of performing experiments}

In this study, a photocatalytic process in an open channel was performed to remove ibuprofen from the wastewater containing this drug. The feed containing medicinal wastewater made with a certain concentration of ibuprofen and certain amounts of photocatalyst of $\mathrm{FeO} / \mathrm{MIC}$ was tested at the mentioned channel at different times (the open channel was placed inside a dark box and solar lamps were installed inside the box). The feed solution was poured into a tank which entered the setup using a centrifugal pump and was exposed to the light of solar lamps $\left(300 \mathrm{~W} / \mathrm{m}^{2}\right)$. In order to increase the mixing between the wastewater and the photocatalyst, 18 mixers were placed in different parts of the channel. After the passage of a specified period of time, the treated sample was taken out of the setup and after the removal of the magnetic photocatalyst from the treated wastewater by a magnet, the final sample was prepared to determine the rate of ibuprofen removal. The schematic of the setup prepared for all the experiments is presented in Figure 1.

A UV-visible spectrophotometer (PG Instruments, England, Model: T80 ++) was used to measure the concentration of the drug (ibuprofen). The calibration curve of the device was drawn separately by preparing solutions with different concentrations of ibuprofen. The studied variables included the initial concentration of ibuprofen, process time, and the photocatalyst concentration (FeO/MIC). The rate of ibuprofen removal was calculated using Equation 1:

$$
\text { Ibuprofen removal } \%=\frac{C_{d, i}-C_{d, t}}{C_{d, i}} \times 100
$$

where $C_{d, i}$ is the initial concentration of ibuprofen in $\mathrm{mg} / \mathrm{L}$ and $C_{d, t}$ is the concentration of ibuprofen at time $\mathrm{t}$ in $\mathrm{mg} / \mathrm{L}$.

\subsection{Photocatalyst (FeO/MIC) characterization}

XRD, SEM, EDAX and FT-IR analyzes were performed to identify the photocatalyst (iron oxide supported on modified Iranian clinoptilolite) as well as the modified Iranian clinoptilolite prepared in this study. XRD pattern of the samples was prepared by a Philips X-ray diffractometer model PW 1730 (Anode material of $\mathrm{Cu}$ and ). Data intensities were plotted in terms of $2 \mathrm{q}$ in a range of $10.25-79.95^{\circ}$ with a pace of $0.05^{\circ}$. 
The surface morphology and surface particle size of the samples were obtained via SEM analysis (MIRA3 TESCAN). In addition, energy scattering and quantitative analysis of photocatalyst were performed via EDAX analysis using SEM device. FT-IR spectroscopy of the samples was performed by Thermo Nicolet AVATAR 360 FTIR to determine the functional groups in each sample.

\subsection{Photocatalyst recovery}

Because of cost savings and environmental issues, the recovery of the catalysts used in various processes is of great importance. In this study, the photocatalyst was recovered under optimal operating conditions. In the recovery process, the magnetic photocatalyst (FeO/MIC) was first separated from the refined sample by means of a strong magnet. The isolated photocatalyst was then washed several times with deionized water to remove impurities and after drying was used again as a photocatalyst in the next step.

\section{Results And Discussion}

\subsection{Photocatalyst characterization}

XRD analysis was used to determine the shape of the crystals in the photocatalyst and its support. Figure 2 presents XRD diagrams of modified Iranian clinoptilolite and iron oxide supported on modified Iranian clinoptilolite. As shown in this figure, there are a large number of peaks in the diagram of the modified Iranian clinoptilolite that indicate the presence of silicon oxide crystals (JCPDS card no. 01-076-0939 and JCPDS card no. 00-044-0696). The peaks for JCPDS card no 01-076-0939 in 2q are equal to 22.1, 28.5, 36.4 , and 65.3 and for JCPDS card no $00-044-0696$ in $2 q$ are equal to $10.3,11.6,17.6,22.8$, and 23.2. In addition, calcium silicate crystals (JCPDS card no 01-087-1260) in $2 q$ are equal to $30.5,32.3$ and 57.6 and calcium magnesium silicate crystals (JCPDS card no 01-083-1819) in $2 q$ are equal to 13.6, 26.5, $30.5,49.2,62.5$, and 74.9. With loading ferrous sulfate heptahydrate on the modified Iranian clinoptilolite, the crystals of iron sulfate (JCPDS card no 01-073-0148) and sodium aluminum silicate (JCPDS card no 01-076-0898) at $2 \mathrm{q}$ are simultaneously equal to $14.9,20.3,21.8,23.8,24.7,26.7,29.8,31.3,32.6,36.0$, $42.7,47.0,50.8,53.5,59.2,61.8,64.8$ and 77.4. XRD diagrams show a similar trend in the peaks in the $\mathrm{MIC}$ and the $\mathrm{FeO} / \mathrm{MIC}$ photocatalyst, and new peaks are formed due to the presence of $\mathrm{Fe}$ and $\mathrm{Fe}^{2+}$ in the $\mathrm{FeO} / \mathrm{MIC}$ photocatalyst.

SEM analysis was used to evaluate the morphological and surface characteristics of $\mathrm{MIC}$ and $\mathrm{FeO} / \mathrm{MIC}$ (Figure 3). As presented in Figure 3-a, MIC crystals have layered or a layer-like structure. The SEM image of the FeO/MIC sample (Figure 3-b) also presents the same MIC structure. As a result, MIC crystals are not affected by $\mathrm{Fe}^{2+}$ and $\mathrm{FeO}$ loading.

The EDAX spectrum of the FeO/MIC sample is also presented in Figure 4. Based on the results of EDAX analysis, the percentage of elements were C: 3.45 wt. \%, 0: 52.80 wt. \%, Na: 0.18 wt. \%, Mg: 0.29 wt. \%, Al: 2.38wt. \%, Si: 23.56 wt. \%, S: 6.64 wt. \%, K: 0.59 wt. \% and Fe: 10.11 wt. \%. 
The analysis of FT-IR spectra is a very important technique for determining the characteristics of functional groups as well as the changes of these groups in photocatalysts. FT-IR spectrum of the modified Iranian clinoptilolite is presented in Figure 5-a and the spectrum of iron oxide supported on modified Iranian clinoptilolite is presented in Figure 5-b.

Figure 5 presents the main groups at peaks of $471,611,798,952,1090,1632$, and $3438 \mathrm{~cm}^{-1}$ in the MIC. The group at $472 \mathrm{~cm}^{-1}$ is related to the stretching vibrations of the Al-O bond, while the peaks at 798 and $1090 \mathrm{~cm}^{-1}$ are related to the Si-O-Si bond (Chávez et al. 2010). Vibration bonds located at 1632 and 3438 $\mathrm{cm}^{-1}$ indicate the tensile frequency of the hydroxyl functional group (OH) (Han et al. 2010). Figure 5 also presents the FT-IR spectrum of the FeO/MIC sample used to investigate changes due to the presence of $\mathrm{FeO}$ in the modified Iranian clinoptilolite. Breck showed that with inserting metal cations into a zeolite structure, some minor changes may occur in the position of the peaks located to the right of the tensile bond of TOT or OTO ( $\mathrm{T}=\mathrm{Al}$ or Si) (Breck 1984). Minor variations in peaks can be observed at 475, 623, $794,1112,1637$, and $3455 \mathrm{~cm}^{-1}$ in the $\mathrm{FeO} / \mathrm{MIC}$ spectrum. There is also a peak at $661 \mathrm{~cm}^{-1}$, indicating the presence of $\mathrm{FeO}$ in the MIC structure. Similar results have been observed in previous studies that used a combination of some metal cations and sulfides or oxides related to different zeolites (NezamzadehEjhieh and Khorsandi 2010; Nezamzadeh-Ejhieh and Hushmandrad 2010; Nezamzadeh-Ejhieh and Salimi 2010).

\subsection{Effect of operational variables on ibuprofen in the wastewater}

In this study, the effect of different variables including the initial ibuprofen concentration, photocatalyst concentration (FeO/MIC), and process time on the removal of ibuprofen in the wastewater was investigated. The initial concentration of ibuprofen $\left(C_{d, i}\right)$ was studied in the range of $5-25 \mathrm{mg} / \mathrm{L}$, the concentration of photocatalyst $\left(C_{\mathrm{FeO} / \mathrm{MIC}}\right)$ in the rang of $100-300 \mathrm{~g} / \mathrm{L}$, and the process time $(\mathrm{t})$ in the range of 10-240 min.

Figure 6 shows the percentage curves of ibuprofen removal over time at different photocatalyst concentrations (FeO/MIC). As shown in the diagrams in this figure, the amount of ibuprofen in the wastewater decreased over time. With the passage of time up to $120 \mathrm{~min}$, the slope of ibuprofen removal decreased, but the overall percentage of ibuprofen removal increased. At $150 \mathrm{~min}$, the percentage of ibuprofen removal was almost constant over time, but until the end of $240 \mathrm{~min}$, the dye removal rate was slightly increased.

As can be observed in the diagrams in Figure 6, the concentration of the photocatalyst plays an important role in the rate of ibuprofen removal. With increasing the concentration of the photocatalyst from 100 $\mathrm{mg} / \mathrm{L}$ to $300 \mathrm{mg} / \mathrm{L}$, there was an increasing trend in the removal of ibuprofen in the wastewater containing this drug. In addition, comparing Figures 6-a, 6-b, and 6-c, it is clear that with increasing the initial concentration from $5 \mathrm{mg} / \mathrm{L}$ to $15 \mathrm{mg} / \mathrm{L}$ (at all the photocatalyst concentrations), there was a relatively proper increasing trend in the removal of ibuprofen from the wastewater containing this drug. With increasing the initial concentration of ibuprofen from $15 \mathrm{mg} / \mathrm{L}$ to $25 \mathrm{mg} / \mathrm{L}$, the increasing trend of 
ibuprofen removal continued, but the rate of increase in ibuprofen removal was lower than the state when the initial concentration changed from $5 \mathrm{mg} / \mathrm{L}$ to $15 \mathrm{mg} / \mathrm{L}$.

According to the results presented in Figures 6-a, 6-b and 6-c, the initial concentration of $25 \mathrm{mg} / \mathrm{L}$, photocatalyst concentration of $300 \mathrm{mg} / \mathrm{L}$, and the process time of $210 \mathrm{~min}$ can be introduced as the optimal operating conditions. Under the mentioned condition, the removal rate of ibuprofen is $99.80 \%$.

\subsection{Kinetic modeling of photocatalytic process}

Normally, the kinetics of photocatalytic reactions are consistent with the Langmuir-Hinshelwood model (Faramarzpour et al. 2009; Zhang et al. 2012):

$$
r=\frac{d C}{d t}=\frac{k K C}{1+K C}
$$

where is the pollutant removal rate in $\mathrm{mg} /(\mathrm{L} . \mathrm{min})$ after the time $t$ in $\mathrm{min}, C$ is the pollutant concentration in $\mathrm{mg} / \mathrm{L}, k$ is the reaction rate constant in $\mathrm{mg} /(\mathrm{L} . \mathrm{min})$, and is the constant of Langmuir-Hinshelwood absorption equilibrium in $\mathrm{L} / \mathrm{mg}$. When the initial concentration $\left(C_{0}\right)$ is about $\mathrm{mM}$ ( $C_{0}$ is very small), the equation can be simplified to a quasi-first-order equation as follows:

$$
r=\frac{d C}{d t}=k K C \rightarrow \ln \left(\frac{C_{0}}{C}\right)=k K t=k^{\prime} t
$$

where $k^{\prime}=k K$ in $\min ^{-1}$ is a quasi-first-order reaction constant, and its value is obtained through plotting the diagram of $\left(\frac{C_{0}}{c}\right)$ in terms of time.

Figure 7 indicates that $\left(\frac{C_{0}}{c}\right)$ has a linear relationship with $\mathrm{t}$, which is proved by a high correlation coefficient $\left(R^{2}>0.95\right)$. Table 2 presents the constant values of quasi-first-order reaction $(k)$ for different conditions (different values of the initial ibuprofen concentration and the photocatalyst concentration) in the ibuprofen removal photocatalytic reaction. As shown in this table, with increasing the initial concentration of ibuprofen as well as increasing the photocatalyst used, the value of the constant of reaction increases. A similar trend was observed in a study by Mousavi Mortazavi et al. that investigated the elimination of furfural (Mousavi-Mortazavi and Nezamzadeh-Ejhieh 2016).

\subsection{Recovery and reusability of FeO/MIC}

The process of photocatalyst recovery was performed according to the mentioned method under optimal conditions (the initial ibuprofen concentration of $25 \mathrm{mg} / \mathrm{L}$ and the adsorbent concentration of $300 \mathrm{mg} / \mathrm{l}$ and the process time of $210 \mathrm{~min}$ ). In order to recover the magnetic photocatalyst (FeO/MIC), a strong magnet was used first to separate $\mathrm{FeO} / \mathrm{MIC}$ from the refined sample. The isolated $\mathrm{FeO} / \mathrm{MIC}$ was then washed several times with deionized water and after drying, it was used again as a photocatalyst in the 
next step. Figure 8 presents a summary of the results of tests on photocatalyst recovery. As presented in this figure (Figure 8), after re-use of the photocatalyst for five times, the removal rate of ibuprofen was still above $91 \%$, and finally, in the sixth and seventh re-use, the ibuprofen removal rate was decreased to about $83 \%$ and $70 \%$, respectively. These results indicate the high potential of the photocatalyst used in this study during the recovery process.

\section{Conclusion}

The removal of pharmaceutical contaminants from wastewater is one of the topics that has been investigated in many studies. In this study, iron oxide supported on modified Iranian clinoptilolite was used as a photocatalyst in the presence of a solar lamp to remove ibuprofen from the wastewater containing this drug. The effect of the initial ibuprofen concentration, the photocatalyst concentration (FeO/MIC), and the process time were investigated as three important parameters involved in this process. The results indicated that the photocatalyst used had a high potential for removing ibuprofen. Furthermore, in this study, a quasi-first-order kinetic model was investigated for modeling and the results showed that the proposed model had a satisfactory level of accuracy. Finally, the photocatalyst was recovered under optimal operating conditions, and after reusing the photocatalyst for five times, the rate of ibuprofen removal from the wastewater containing this drug was about $91 \%$.

\section{Declarations}

\section{Ethics approval and consent to participate}

Not applicable.

\section{Declaration of competing interest}

The authors declare that they have no known competing financial interests or personal relationships that could have appeared to influence the work reported in this paper.

\section{Funding}

The authors did not receive support from any organization for the submitted work.

\section{Authors' contributions}

Majid Mohadesi: Supervision, Methodology, Software, Writing- Reviewing and Editing

Ashkan Gouran: Data curation, Writing- Original draft preparation, Investigation

Kiarash Seifi: Data curation, Writing- Original draft preparation

\section{Availability of data and materials}


All data, models, or code generated or used during the study are available in a repository or online.

\section{Consent for publication}

Not applicable.

\section{References}

1. Aghel, B., Mohadesi, M., Gouran, A., \& Razmegir, M. H. (2020). Use of modified Iranian clinoptilolite zeolite for cadmium and lead removal from oil refinery wastewater. International Journal of Environmental Science and Technology, 17(3), 1239-1250.

2. Apopei, P., Orha, C., Popescu, M. I., Lazau, C., Manea, F., Catrinescu, C., \& Teodosiu, C. (2020). Diclofenac removal from water by photocatalysis-assisted filtration using activated carbon modified with $\mathrm{N}$-doped $\mathrm{TiO}_{2}$. Process Safety and Environmental Protection, 138, 324-336.

3. Arthur, R. B., Bonin, J. L., Ardill, L. P., Rourk, E. J., Patterson, H. H., \& Stemmler, E. A. (2018). Photocatalytic degradation of ibuprofen over $\mathrm{BiOCl}$ nanosheets with identification of intermediates. Journal of hazardous materials, 358, 1-9.

4. Breck, D. W. (1984). Zeolite molecular sieves: structure, chemistry and use. Krieger.

5. Candido, J. P., Andrade, S. J., Fonseca, A. L., Silva, F. S., Silva, M. R., \& Kondo, M. M. (2016). Ibuprofen removal by heterogeneous photocatalysis and ecotoxicological evaluation of the treated solutions. Environmental Science and Pollution Research, 23(19), 19911-19920.

6. Chávez, M. L., De Pablo, L., \& García, T. A. (2010). Adsorption of $\mathrm{Ba}^{2+}$ by Ca-exchange clinoptilolite tuff and montmorillonite clay. Journal of Hazardous Materials, 175(1-3), 216-223.

7. Faramarzpour, M., Vossoughi, M., \& Borghei, M. (2009). Photocatalytic degradation of furfural by titania nanoparticles in a floating-bed photoreactor. Chemical Engineering Journal, 146(1), 79-85.

8. Gopinath, K. P., Madhav, N. V., Krishnan, A., Malolan, R., \& Rangarajan, G. (2020). Present applications of titanium dioxide for the photocatalytic removal of pollutants from water: A review. Journal of Environmental Management, 270, 110906.

9. Gu, Y., Yperman, J., Carleer, R., D'Haen, J., Maggen, J., Vanderheyden, S., ... \& Garcia, R. M. (2019). Adsorption and photocatalytic removal of Ibuprofen by activated carbon impregnated with $\mathrm{TiO} 2$ by UV-Vis monitoring. Chemosphere, 217, 724-731.

10. Han, R., Zhang, L., Song, C., Zhang, M., Zhu, H., \& Zhang, L. (2010). Characterization of modified wheat straw, kinetic and equilibrium study about copper ion and methylene blue adsorption in batch mode. Carbohydrate Polymers, 79(4), 1140-1149.

11. Jallouli, N., Pastrana-Martínez, L. M., Ribeiro, A. R., Moreira, N. F., Faria, J. L., Hentati, O., ... \& Ksibi, M. (2018). Heterogeneous photocatalytic degradation of ibuprofen in ultrapure water, municipal and pharmaceutical industry wastewaters using a $\mathrm{TiO}_{2} / \mathrm{UV}$-LED system. Chemical Engineering Journal, 334, 976-984. 
12. Kang, K., Jang, M., Cui, M., Qiu, P., Park, B., Snyder, S. A., \& Khim, J. (2014). Preparation and characterization of magnetic-core titanium dioxide: implications for photocatalytic removal of ibuprofen. Journal of Molecular Catalysis A: Chemical, 390, 178-186.

13. Kumar, A., Khan, M., Zeng, X., \& Lo, I. M. (2018). Development of g- $\mathrm{C}_{3} \mathrm{~N}_{4} / \mathrm{TiO}_{2} / \mathrm{Fe}_{3} \mathrm{O}_{4} @ \mathrm{SiO}_{2}$ heterojunction via sol-gel route: a magnetically recyclable direct contact Z-scheme nanophotocatalyst for enhanced photocatalytic removal of ibuprofen from real sewage wastewater under visible light. Chemical Engineering Journal, 353, 645-656.

14. Li, F., Kang, Y., Chen, M., Liu, G., Lv, W., Yao, K., ... \& Huang, H. (2016). Photocatalytic degradation and removal mechanism of ibuprofen via monoclinic $\mathrm{BiVO}_{4}$ under simulated solar light. Chemosphere, $150,139-144$.

15. Lin, L., Jiang, W., Bechelany, M., Nasr, M., Jarvis, J., Schaub, T., ... \& Xu, P. (2019). Adsorption and photocatalytic oxidation of ibuprofen using nanocomposites of $\mathrm{TiO}_{2}$ nanofibers combined with $\mathrm{BN}$ nanosheets: Degradation products and mechanisms. Chemosphere, 220, 921-929.

16. Liu, F., Liang, J., Chen, L., Tong, M., \& Liu, W. (2019). Photocatalytic removal of diclofenac by Ti doped BiOI microspheres under visible light irradiation: kinetics, mechanism, and pathways. Journal of Molecular Liquids, 275, 807-814.

17. Mousavi-Mortazavi, S., \& Nezamzadeh-Ejhieh, A. (2016). Supported iron oxide onto an Iranian clinoptilolite as a heterogeneous catalyst for photodegradation of furfural in a wastewater sample. Desalination and Water Treatment, 57(23), 10802-10814.

18. Nezamzadeh-Ejhieh, A., \& Hushmandrad, S. (2010). Solar photodecolorization of methylene blue by $\mathrm{CuO/X}$ zeolite as a heterogeneous catalyst. Applied Catalysis A: General, 388(1-2), 149-159.

19. Nezamzadeh-Ejhieh, A., \& Khorsandi, M. (2010). Heterogeneous photodecolorization of Eriochrome Black T using Ni/P zeolite catalyst. Desalination, 262(1-3), 79-85.

20. Nezamzadeh-Ejhieh, A., \& Salimi, Z. (2010). Heterogeneous photodegradation catalysis of ophenylenediamine using CuO/X zeolite. Applied Catalysis A: General, 390(1-2), 110-118.

21. sarafraz, M., Amini, M. M., Adiban, M., \& Eslami, A. (2020). Facile synthesis of mesoporous black N$\mathrm{TiO}_{2}$ photocatalyst for efficient charge separation and the visible-driven photocatalytic mechanism of ibuprofen degradation. Materials Science in Semiconductor Processing, 120, 105258.

22. Tanveer, M., Guyer, G. T., \& Abbas, G. (2019). Photocatalytic degradation of ibuprofen in water using $\mathrm{TiO}_{2}$ and $\mathrm{ZnO}$ under artificial UV and solar irradiation. Water Environment Research, 91(9), 822-829.

23. Xia, D., \& Lo, I. M. (2016). Synthesis of magnetically separable $\mathrm{Bi}_{2} \mathrm{O}_{4} / \mathrm{Fe}_{3} \mathrm{O}_{4}$ hybrid nanocomposites with enhanced photocatalytic removal of ibuprofen under visible light irradiation. Water research, 100, 393-404.

24. Yao, X., Hu, X., Liu, Y., Wang, X., Hong, X., Chen, X., ... \& Wang, D. (2020). Simultaneous photocatalytic degradation of ibuprofen and $\mathrm{H}_{2}$ evolution over Au/sheaf-like $\mathrm{TiO}_{2}$ mesocrystals. Chemosphere, 261, 127759. 
25. Zhang, Y., Wu, H., Zhang, J., Wang, H., \& Lu, W. (2012). Enhanced photodegradation of pentachlorophenol by single and mixed cationic and nonionic surfactants. Journal of hazardous materials, 221, 92-99.

26. Zhang, P., Lu, X. F., Luan, D., \& Lou, X. W. (2020). Fabrication of heterostructured $\mathrm{Fe}_{2} \mathrm{TiO}_{5}-\mathrm{TiO}_{2}$ nanocages with enhanced photoelectrochemical performance for solar energy conversion. Angewandte Chemie, 132(21), 8205-8209.

27. Zhou, A., Liao, L., Wu, X., Yang, K., Li, C., Chen, W., \& Xie, P. (2020). Fabrication of a Z-scheme nanocomposite photocatalyst for enhanced photocatalytic degradation of ibuprofen under visible light irradiation. Separation and Purification Technology, 250, 117241.

\section{Tables}

Table 1. Composition and physicochemical characteristics of ibuprofen used in this study.

\begin{tabular}{|llllll|}
\hline $\begin{array}{l}\text { Drug } \\
\text { name }\end{array}$ & $\begin{array}{l}\text { Chemical } \\
\text { formula }\end{array}$ & Molecular structure & $\begin{array}{l}\mathbf{M}, \\
\mathbf{g} / \mathbf{m o l}\end{array}$ & $\begin{array}{l}\mathbf{\Lambda}_{\text {max }}, \\
\mathbf{n m}\end{array}$ & $\begin{array}{l}\text { Solubility in water, } \\
\mathbf{g} / \mathbf{l}\end{array}$ \\
\hline Ibuprofen & $\mathrm{C}_{13} \mathrm{H}_{18} \mathrm{O}_{2}$ & & & & \\
\end{tabular}

Table 2. Ibuprofen removal reaction rate constant as a function of the initial concentration of ibuprofen and $\mathrm{FeO} / \mathrm{MIC}$ photocatalyst.

\begin{tabular}{llll}
$\boldsymbol{C}_{d, i \boldsymbol{i}} \mathrm{mg} / \mathrm{L}$ & $\mathrm{C}_{\mathrm{FeO} / \mathrm{MIC}}, \mathrm{mg} / \mathrm{L}$ & $\boldsymbol{k}^{\prime} \times 10^{2}, \mathbf{m i n}^{-1}$ & $R^{2}$ \\
\hline 5 & 100 & 1.51 & 0.9744 \\
\hline 5 & 200 & 1.82 & 0.9752 \\
\hline 5 & 300 & 2.21 & 0.9746 \\
\hline 15 & 100 & 1.69 & 0.9513 \\
\hline 15 & 200 & 2.13 & 0.9674 \\
\hline 15 & 300 & 2.89 & 0.9868 \\
\hline 25 & 100 & 1.72 & 0.9710 \\
\hline 25 & 200 & 2.30 & 0.9871 \\
\hline 25 & 300 & 2.86 & 0.9899
\end{tabular}


Figures

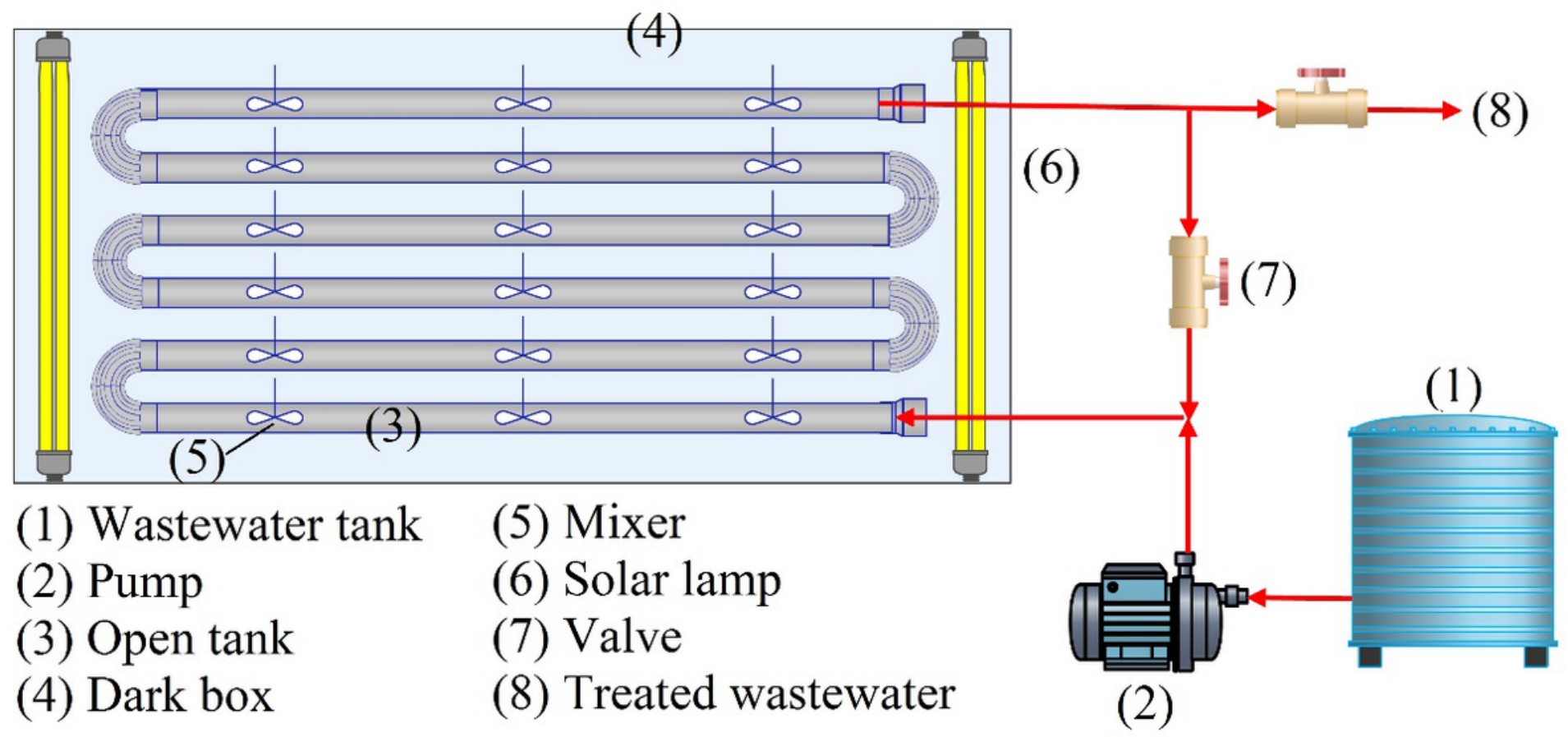

Figure 1

Schematic of the setup used in this study to remove ibuprofen through the photocatalytic process using $\mathrm{FeO} / \mathrm{MIC}$.

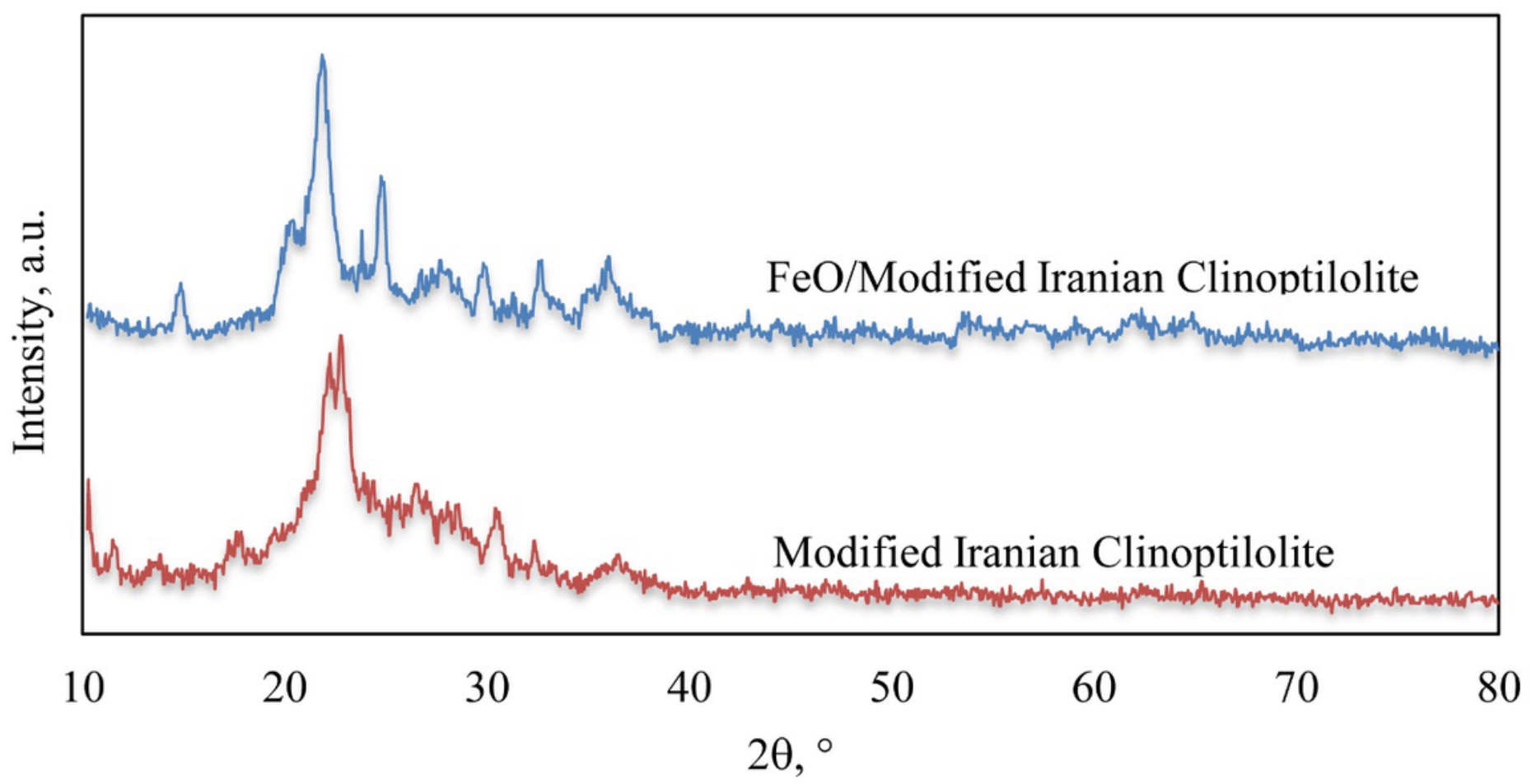

Figure 2 
XRD patterns of MIC and FeO/MIC samples.
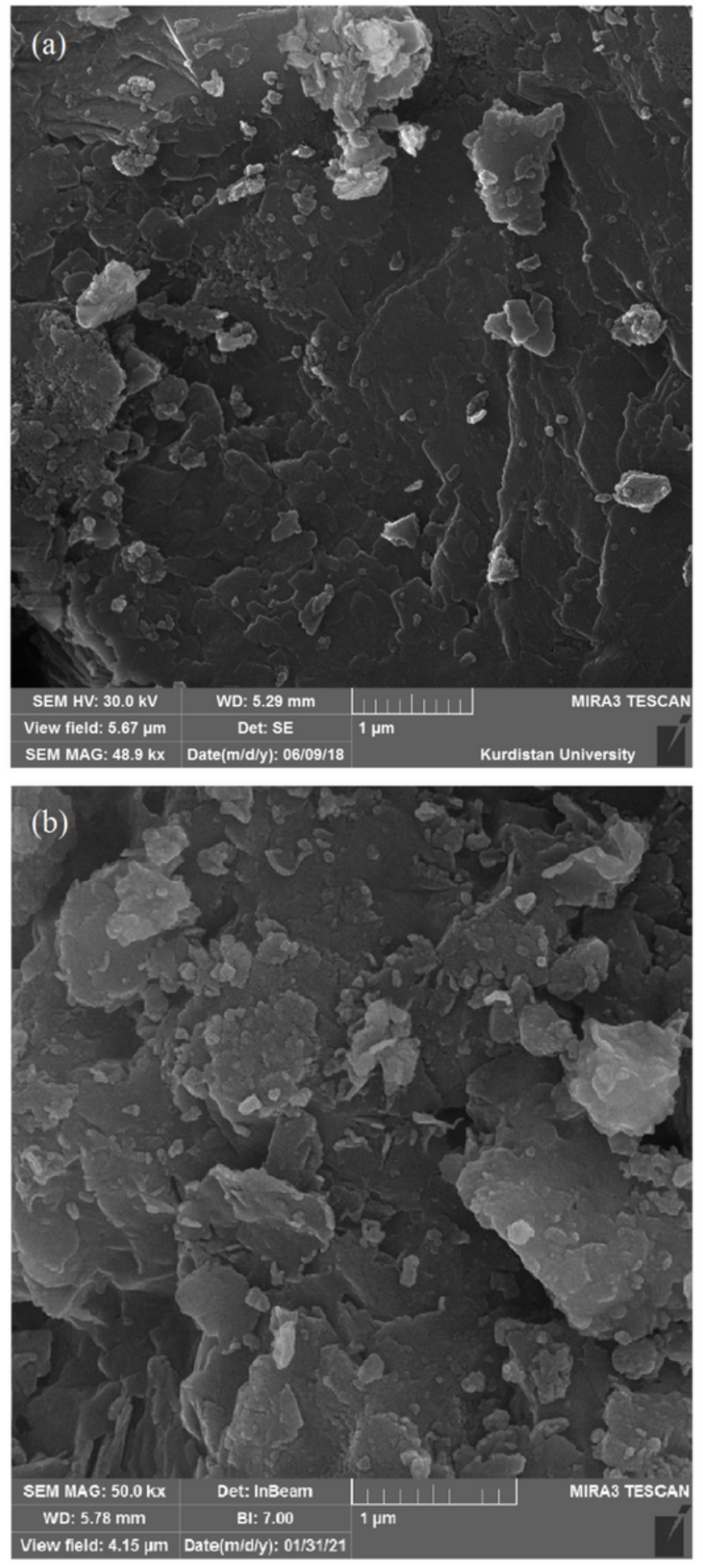

\section{Figure 3}

Scanning electron microscopy (SEM) images; (a) MIC, (b) FeO/MIC. 


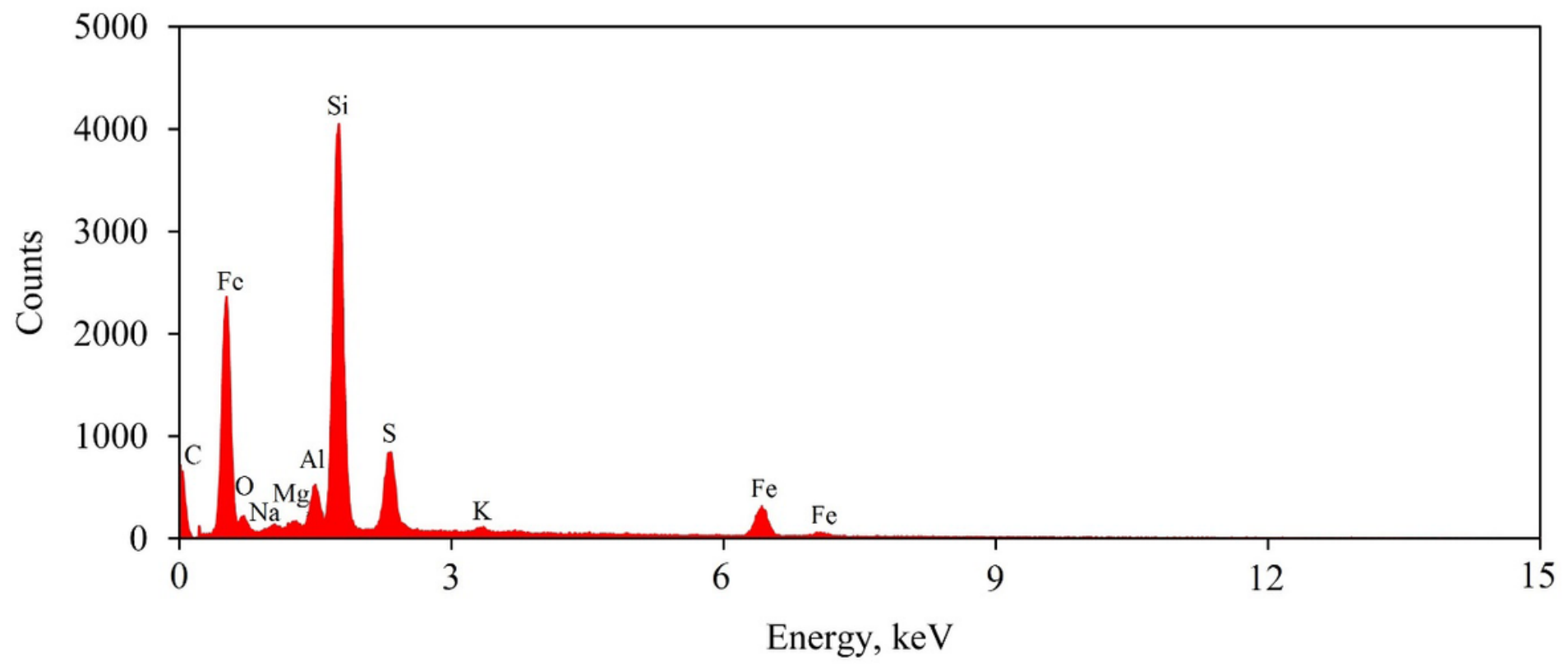

Figure 4

EDAX spectrum for $\mathrm{FeO} / \mathrm{MIC}$.

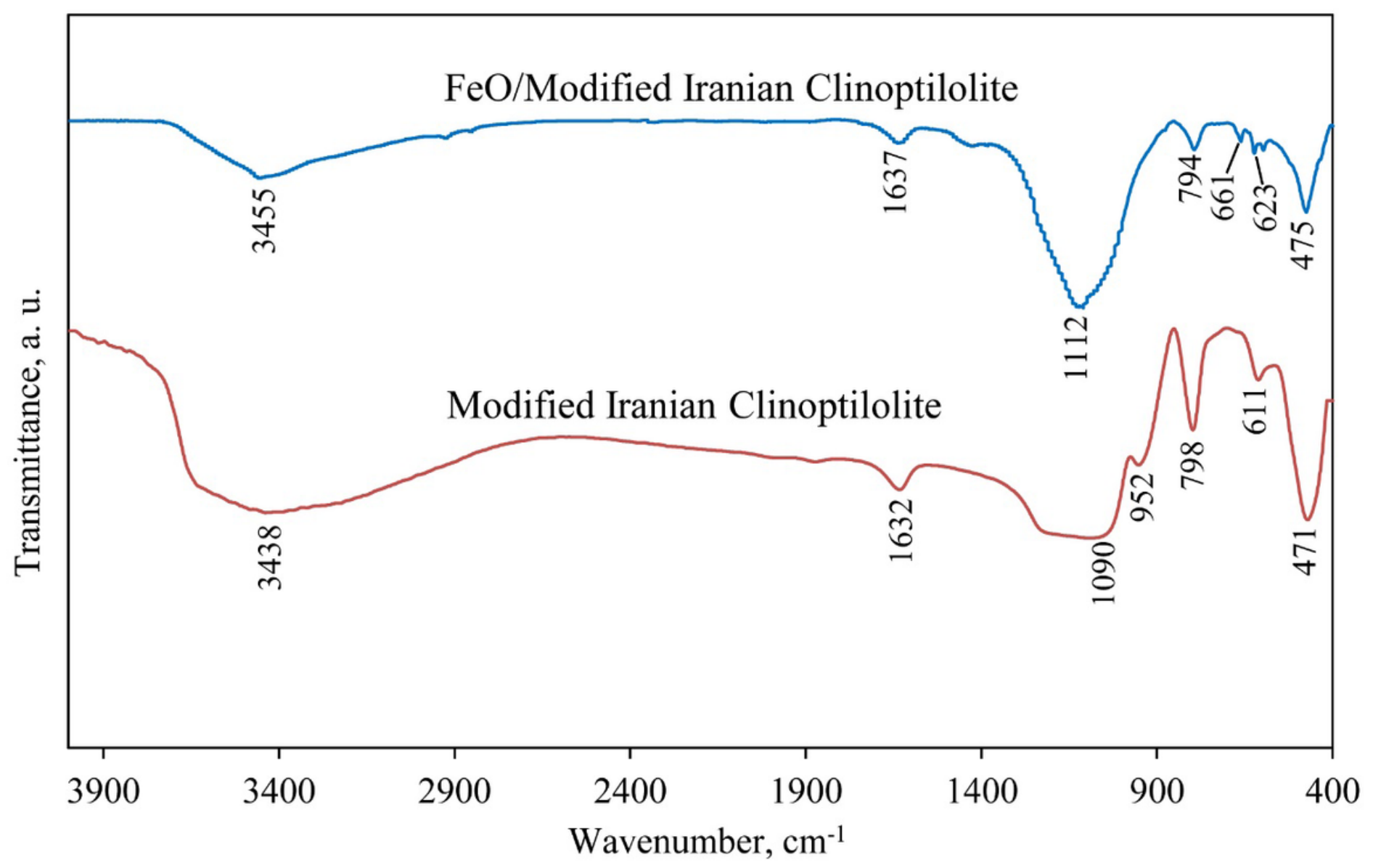

Figure 5 
FT-IR spectrum for MIC and FeO/MIC.
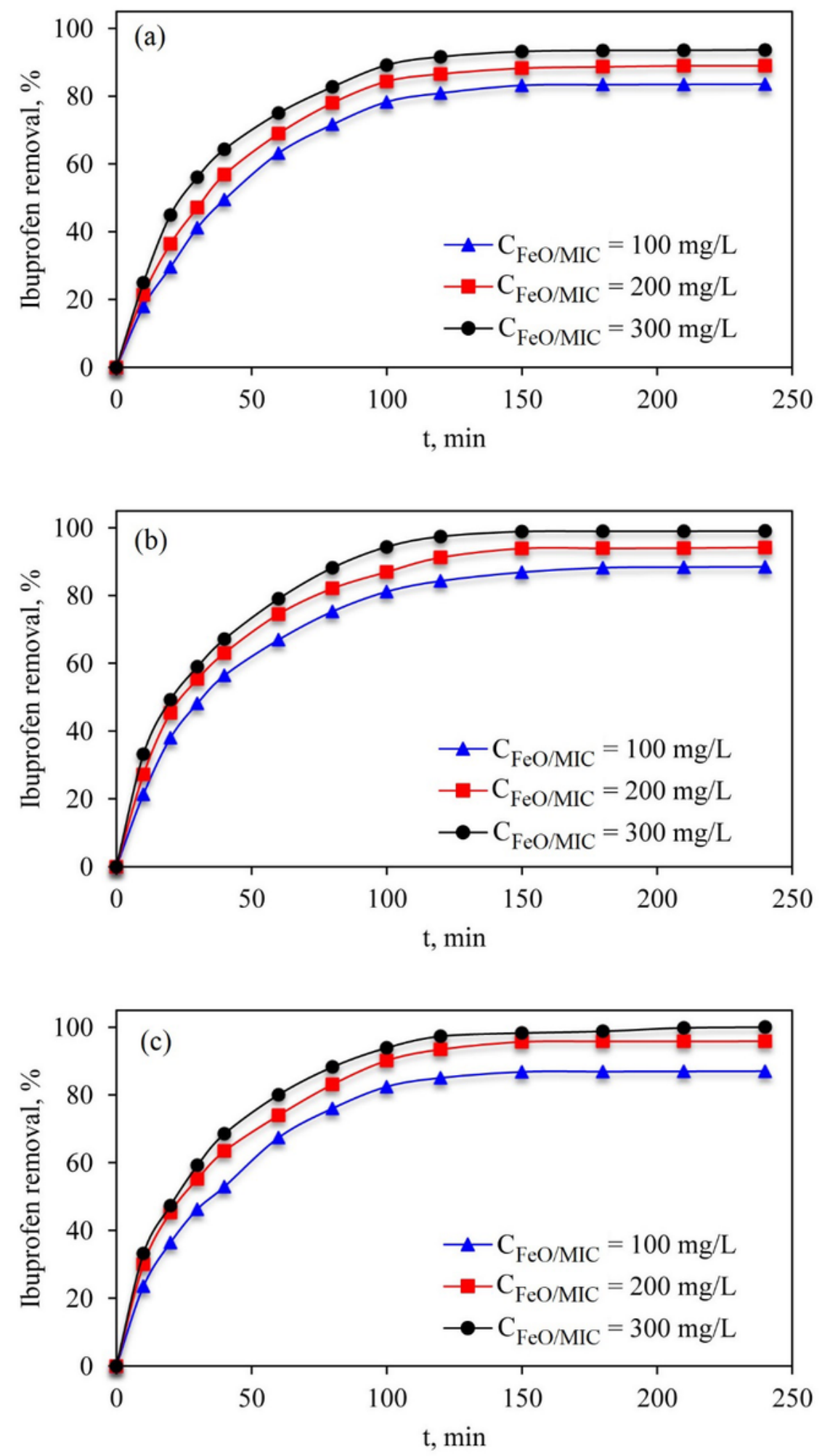

Figure 6

Diagrams of Ibuprofen removal over time at different concentrations of $\mathrm{FeO} / \mathrm{MIC}$ photocatalyst; a) Initial ibuprofen concentration of $5 \mathrm{mg} / \mathrm{L}, \mathrm{b}$ ) Initial ibuprofen concentration of $15 \mathrm{mg} / \mathrm{L}, \mathrm{C}$ ) Initial concentration ibuprofen of $25 \mathrm{mg} / \mathrm{L}$. 

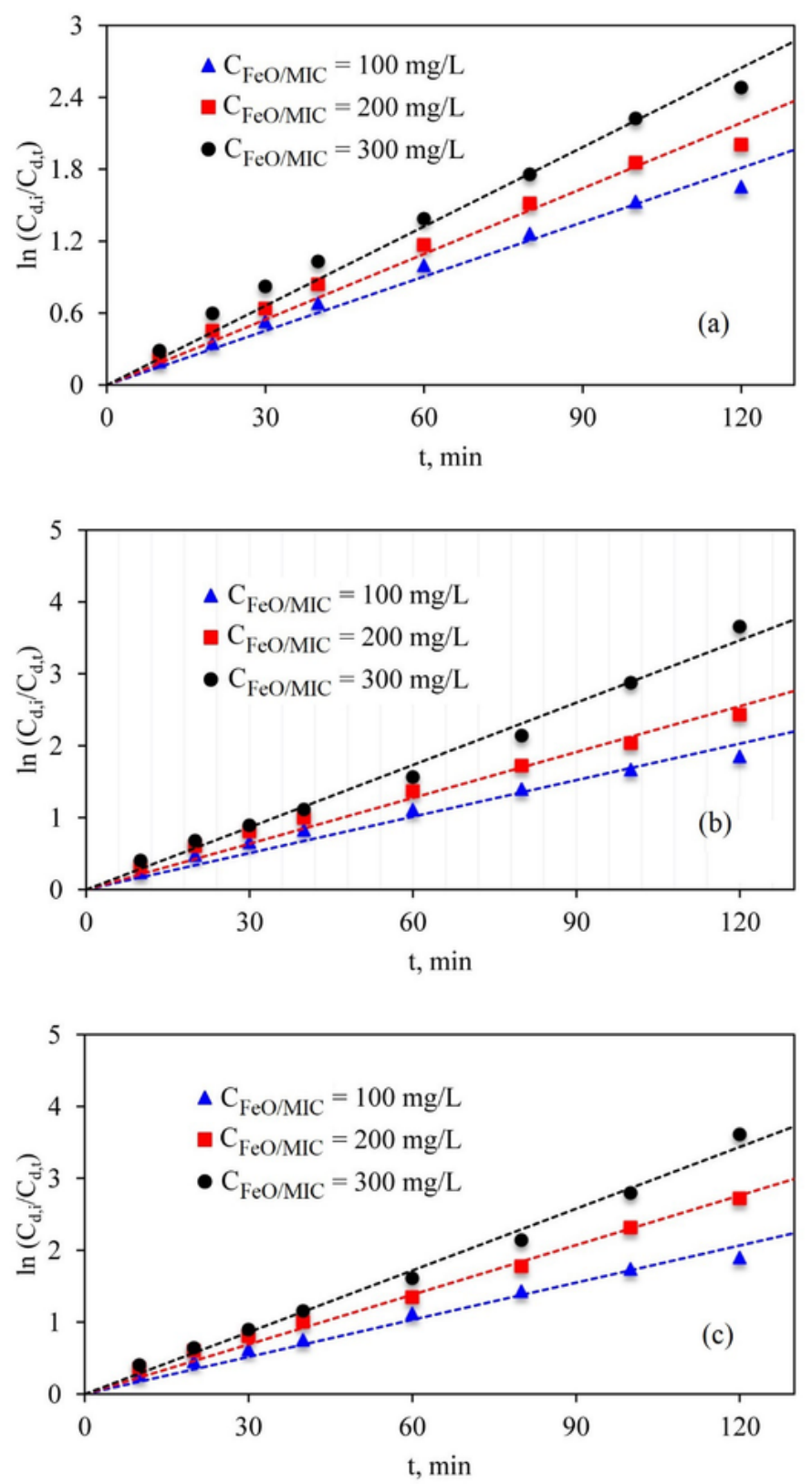

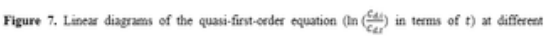

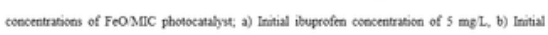
ibuprofen concertation of $15 \mathrm{mg} \mathrm{L}, \mathrm{c}$ ) Irtitial ibeprofen concentration of $25 \mathrm{mg} \mathrm{L}$.

\section{Figure 7}

See image above for figure legend 


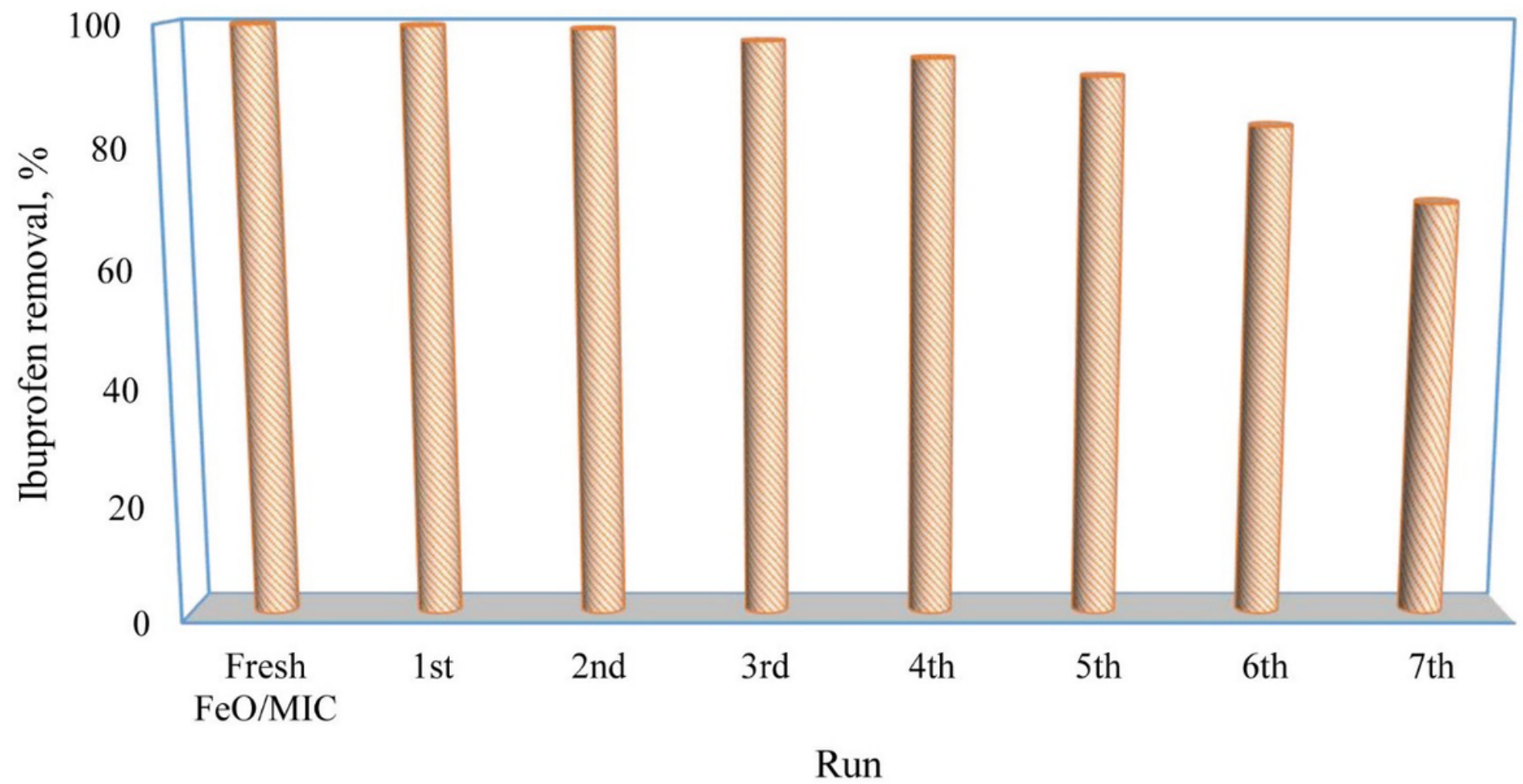

Figure 8

Results of photocatalytic recovery (FeO/MIC) in the process of ibuprofen removal from the wastewater under optimal operating conditions (Cd,i=25 mg/LD CFeO/MIC=300 mg/L and t=210 min). 